\title{
When Cilia Go Bad: The Complex Genetics of Ciliopathies
}

\author{
Anna Lindstrand
}

Disruption of ciliary and basal body function has been associated with a growing number of human genetic disorders, collectively termed ciliopathies. Cilia can roughly be divided into motile or non-motile cilia. Motile cilia can be observed in sperm and airway epithelial cells, while immotile cilia are often referred to as primary cilia and are observed in photoreceptor cells or olfactory neurons. Although clinically distinct, ciliopathies manifest similar hallmark clinical features including retinal degeneration, renal and pancreatic cysts, liver fibrosis, polydactyly and other skeletal abnormalities, situs inversus, male infertility due to defective sperm locomotion or spermatogenic failure, and central and peripheral nervous system defects. More than 30 different ciliopathies have been reported and almost 200 ciliopathyassociated genes are known. Furthermore, genetic studies have indicated that many ciliopathy-associated genes can contribute to different ciliopathy disorders.

Utilizing a custom designed oligonucleotide array-CGH (aCGH) targeting the coding exons of $\sim 2000$ genes of interest we have studied the genetic contribution of copy number variations (CNVs) throughout a spectrum of ciliopathies. In this way, we have identified several novel genes such as ARMC4 underlying Primary Ciliary Dyskinesia (PCD, MIM244400), IFT74 in Bardet-Biedl syndrome (BBS, MIM 209900) and WDR63 in isolated encephalocele. Furthermore, we have shown that CNVs contribute pathogenic alleles to a substantial fraction of affected individuals both as primary disease drivers but also play a critical role in modifying disease penetrance and expressivity.

\footnotetext{
A. Lindstrand $(\bowtie)$

Department of Molecular Medicine and Surgery, Karolinska Institutet, Solna, Sweden

Clinical Genetics, Karolinska University Hospital, Solna, Sweden

e-mail: Anna.Lindstrand@ki.se 